\title{
Association between the preoperative neutrophil-to-lymphocyte ratio and tertiary lymphoid structures surrounding tumor in gastric cancer
}

\author{
YOSHIHITO YAMAKOSHI, HIROAKI TANAKA, CHIE SAKIMURA, TAKUYA MORI, \\ SOTA DEGUCHI, MAMI YOSHII, TATSURO TAMURA, TAKAHIRO TOYOKAWA, \\ SHIGERU LEE, KAZUYA MUGURUMA, KOSEI HIRAKAWA and MASAICHI OHIRA
}

Department of Gastroenterological Surgery, Osaka City University Graduate School of Medicine, Osaka 545-8585, Japan

Received September 10, 2020; Accepted February 12, 2021

DOI: $10.3892 / \operatorname{mco} .2021 .2238$

\begin{abstract}
The neutrophil-to-lymphocyte ratio (NLR) has been reported to be associated with a poor prognosis in various types of cancer. We previously reported that an antitumor immune response was induced by tertiary lymphoid structures (TLSs) surrounding tumor, and increased TLS was an independent prognostic factor in patients with gastric cancer. The present study examined the stratification based on the correlation between the preoperative NLR and TLS density in gastric cancer. A total of 199 patients who underwent surgery for stage Ib-IV gastric cancer were included in the study. Receiver operating characteristic curve analysis was used to determine the appropriate cut-off values of the preoperative NLR and the TLS density. The prognostic factors were evaluated in a multivariate analysis. The median NLR was 2.18 (mean \pm SD, $2.7 \pm 2.04$ ). A total of 91 patients with an NLR $\geq 2.33$ was classified into the high NLR group. The overall survival was significantly improved in patients with a low NLR than in those with a high NLR. Additionally, the low NLR group tended to have a high TLS density. The multivariate analysis indicated that the preoperative NLR and TLS density were independent risk factors. When the patients were classified into the high and low NLR and TLS groups and the survival rates were compared, the prognosis was significantly improved in the low NLR and high TLS group than in the other groups. The preoperative NLR may be associated with the presence of TLSs surrounding the tumor, and the combination of NLR and TLS may be useful for the stratification of patient prognosis. The present results suggested that the NLR and TLS
\end{abstract}

Correspondence to: Dr Hiroaki Tanaka, Department of Gastroenterological Surgery, Osaka City University Graduate School of Medicine, 1-4-3 Asahi-machi, Abeno, Osaka 545-8585, Japan E-mail: hiroakitan@med.osaka-cu.ac.jp

Key words: neutrophil-to-lymphocyte ratio, tertiary lymphoid structure, gastric cancer, tumor-associated neutrophils, tumor infiltrating lymphocytes density may be surrogate markers for immunotherapy against gastric cancer.

\section{Introduction}

Immune checkpoint blockade (ICB) has proven effective for prolonging the prognosis in gastric cancer (1). The main mechanism of action in ICB therapy is the anti-tumor immune response of tumor-infiltrating lymphocytes (TILs) against cancer cells. Many studies have reported that TILs are associated with a good prognosis (2-6). Meanwhile, several studies have reported that tertiary lymphoid structures (TLSs) are associated with favorable clinical outcomes in patients with various types of cancer, including lung, gastric, colorectal and breast cancer (7-10), and we previously reported that TLSs are positively associated with TILs (11). Moreover, recent studies have shown that TLSs might play an important role in sustaining the anti-tumor immune response to ICB therapy $(12,13)$. TLSs are transient ectopic lymphoid organizations that are detected in the invasive margin of the tumor and/or in the stroma of most cancers and display an overall organization similar to that observed in secondary lymphoid organs, such as the lymph nodes (14). TLSs are composed of B-cell follicles, T-cell zones, follicular dendritic cells and high endothelial venules $(15,16)$.

On the other hand, as the tumor grows, non-specific inflammatory responses caused by cancer cells or surrounding tissue tend to increase the peripheral blood neutrophil count and reduce the lymphocyte count (17). Thus, systemic inflammatory responses, including the high neutrophil-to-lymphocyte ratio (NLR), are related to tumor development and progression (18) and have been shown to be associated with outcomes in patients with various malignancies, including esophageal squamous cell carcinoma, gastric cancer, colorectal cancer, and hepatocellular carcinoma (19-23). The NLR could also be useful in the diagnosis of thyroiditis (24) and as an indicator to differentiate malignant from benign thyroid nodules in the preoperative period (25). In addition, it could be associated with glucose control and correlated with the HbA1c level in type 2 diabetes mellitus $(26,27)$, and it could serve as a diagnostic tool for various other inflammatory conditions, such as 
ulcerative colitis, irritable bowel syndrome, and nonalcoholic fatty liver disease (28-30).

According to the above reasons, the NLR can be a useful biomarker for various cancers, and TLSs have a vital role in the anti-tumor immune response, such as in the prevention of tumor progression by increasing the numbers of TILs, and may be an independent prognostic marker in various cancers. Therefore, we hypothesized that the NLR, an indicator of the systemic inflammatory response, might reflect TLSs in the tumor microenvironment, and investigated the association between the preoperative NLR and clinicopathological features and their relevance to the TLS density surrounding the tumor in gastric cancer.

\section{Patients and methods}

Patients. This retrospective study included all 199 consecutive patients with stage IB-IV gastric cancer who had undergone initial surgical resection without preoperative chemotherapy or radiotherapy between 2007 and 2010 at Osaka City University Hospital, Japan. Patients with stage IA disease were excluded from this study because the tumor had been resected by Endoscopic mucosal resection (EMR) or because of the small size of the tumor, which makes it difficult to assess TLSs. All patients were followed up regularly until April 2015 or until their death. Follow-up examinations were scheduled for every three months for the first two years, every six months during the third to fourth years, and annually thereafter. The median follow-up period after surgery was 49 (1-92) months. Overall survival (OS) was defined as the time between the date of surgery and death, and diseasefree survival (DFS) was defined as the time between the date of surgery and recurrence. This study was approved by the Osaka City University Ethics Committee. Informed consent was obtained from all patients.

Datacollection.Clinicopathological information was extracted retrospectively from hospital data. The patient data included age, sex, smoking history, tumor staging (TNM), histological type, lymphatic invasion, venous invasion, preoperative NLR and TLS density. Pathological TNM staging was recorded for all patients based on the UICC TNM classification, 7th edition. The preoperative NLR, which is calculated by dividing the absolute neutrophil count by the absolute lymphocyte count, was determined based on routine test results from peripheral blood samples that were collected within two weeks before the operation. In cases with multiple blood samples, the sample from the first hospital visit was used to calculate the NLR. Then, to examine the impact of the preoperative NLR on the clinicopathological features, we divided the patients into 2 groups according to the NLR, and compared the low and high NLR groups.

Immunohistochemistry. The centers of TLSs surrounding tumor were located in B cells that formed clusters. We therefore counted B cell clusters as TLSs, as we previously reported (11). Immunohistochemistry was performed on 4- $\mu \mathrm{m}$ thick sections from formalin-fixed paraffin-embedded (FFPE) tumor blocks, which were obtained from patients with gastric cancer and fixed with $10 \%$ formalin at room temperature for 6-48 h. After incubation at $60^{\circ} \mathrm{C}$ for $10 \mathrm{~min}$, the sections were deparaffinized using xylene and rehydrated in a graded ethanol series (70, 80, 90 and 100\%) for 3 min each time, twice. Endogenous peroxidase activity was blocked with absolute methanol containing $3 \%$ hydrogen peroxide at room temperature for $15 \mathrm{~min}$. After washing the sections in PBS, they were microwaved for $10 \mathrm{~min}$ to achieve antigen retrieval. Non-specific binding was blocked using the non-specific staining blocking reagent, Target Retrieval Solution (Dako; Agilent Technologies, Inc.), which was diluted 10 times with sterile distilled water, and the samples incubated at $95^{\circ} \mathrm{C}$ for $45 \mathrm{~min}$. The sections were subsequently incubated with the primary antibody overnight at $4^{\circ} \mathrm{C}$, following which, they were incubated with the secondary antibody, histofine reagent (pre-diluted; Nichirei Biosciences, Inc.) at room temperature for $10 \mathrm{~min}$, and the signal was visualized using 3-3'-diaminobenzidine, and finally counter-stained with hematoxylin at room temperature for $20 \mathrm{sec}$ before mounting. The primary antibodies used for the immunohistochemical analyses were mouse anti-CD20 for B cells (clone L26; pre-diluted; Dako; Agilent Technologies, Inc.). The primary antibodies were diluted with 5\% BSA (Sigma-Aldrich, Inc.; Merck KGaA) in PBS. Then, we measured the area $\left(\mathrm{mm}^{2}\right)$ of CD20-positive cells and calculated the CD20-positive area (\%) of each field using the ImageJ software program (version 15.1; National Institutes of Health). The $\mathrm{CD} 2 \mathrm{O}^{+} \mathrm{B}$ cell density was determined as the mean CD20-positive area in three fields. Fig. 1 shows TLS-high and TLS-low images in one low-power field of view.

Statistical analysis. All statistical analyses were performed using the JMP software program (version 13; SAS Institute, Inc.). The receiver operating characteristic (ROC) curve and area under the ROC curve were used to select the best cut-off values for the preoperative NLR and TLS density. Categorical variables were compared using the chi-squared test.Correlation analysis was performed using Pearson's correlation analysis. The Kaplan-Meier method and log-rank test were used to compare survival curves. Univariate and multivariate analyses were performed using a Cox proportional hazards regression model. P-values of $<0.05$ were considered to indicate statistical significance.

\section{Results}

The association of the preoperative NLR with the clinicopathological features. The median and mean values of the preoperative NLR were 2.18 and 2.7, respectively, with a standard deviation (SD) of 2.04 and a range of 0.59-15.17. The ROC analysis showed that the optimal cut-off value of the preoperative NLR was 2.33 (area under the curve [AUC] $0.625)$ (Fig. 2A). Based on the cutoff value, the diagnostic sensitivity and specificity were 66.7 and $61.5 \%$, respectively. This value was then used to divide the patients into 2 groups: The low NLR group $(<2.33 ; n=108)$ and the high NLR group $(\geq 2.33 ; n=91)$. There were no significant differences in age, sex, smoking history, T category, $\mathrm{N}$ category, TNM stage, histological type or incidence of venous invasion between the groups (Table I). The high NLR group had a higher incidence of lymphatic invasion than the low NLR group. The overall survival of the high NLR group was significantly worse than 


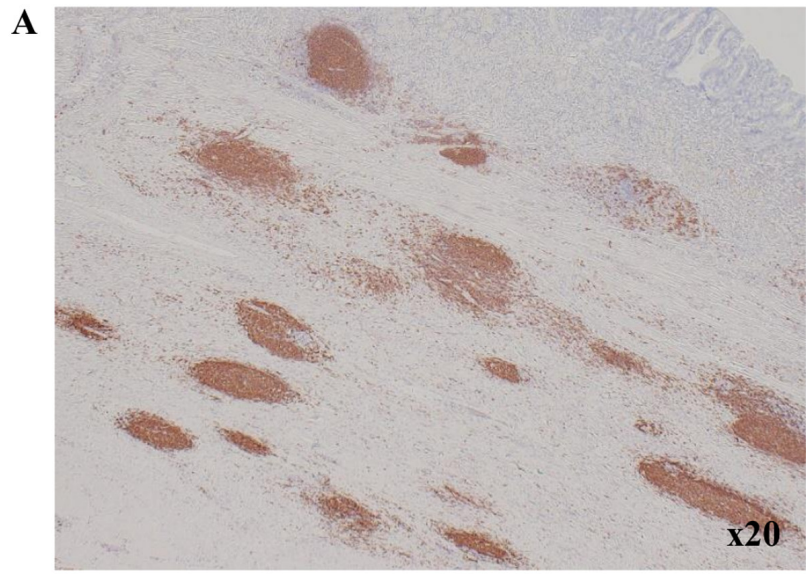

B

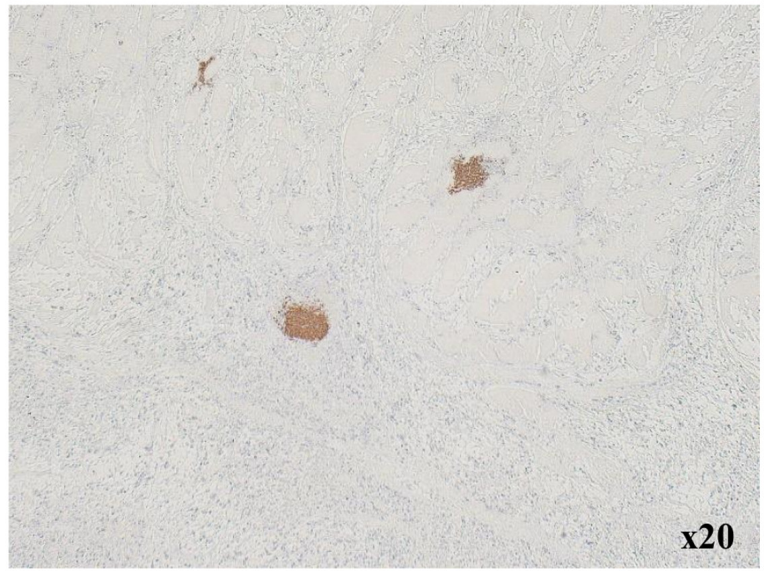

Figure 1. Immunohistochemistry showing TLS surrounding the primary tumor in gastric cancer. (A) TLS high and (B) TLS low (magnification, x20). TLS, tertiary lymphoid structure.

that of the low NLR group (Fig. 3). The 5-year survival rate was $66.9 \%$ in the low NLR group and $39.5 \%$ in the high NLR group.

The association of the TLS with the NLR and the impact on survival. The median TLS density was 1.78 (average $2.96 \pm 2.72$ ). The ROC analysis showed that the optimal cut-off value of the TLS density was 2.00 (AUC 0.619) (Fig. 2B). Based on the cutoff value, the diagnostic sensitivity and specificity were 56.5 and $63.7 \%$, respectively. A scatter chart plotting the preoperative NLR and TLS density revealed a marginal negative association; however, this association did not reach statistical significance $(\mathrm{P}=0.0084, \mathrm{R}=-0.1864)$ (Fig. 4). The comparison of the 2 groups revealed that $66 \%$ of the high NLR group had fewer TLSs, and 58\% of the low NLR group had more TLSs (Table II). Regarding the survival curves according to the combination of the preoperative NLR and TLS density, the OS of the low NLR/high TLS density groups was significantly better than that of the other groups (Fig. 5). In an analysis according to stage, the high NLR/low TLS group also had a significantly worse prognosis than the low NLR/high TLS group (Fig. 6).

Regarding the prognostic factors, the univariate analysis showed that the T stage, N stage, TNM stage, histological type, lymphatic invasion, NLR and TLS density were associated with overall survival. The multivariate analysis results showed that the $\mathrm{T}$ stage, histological type, NLR and TLS density
A Preoperative NLR

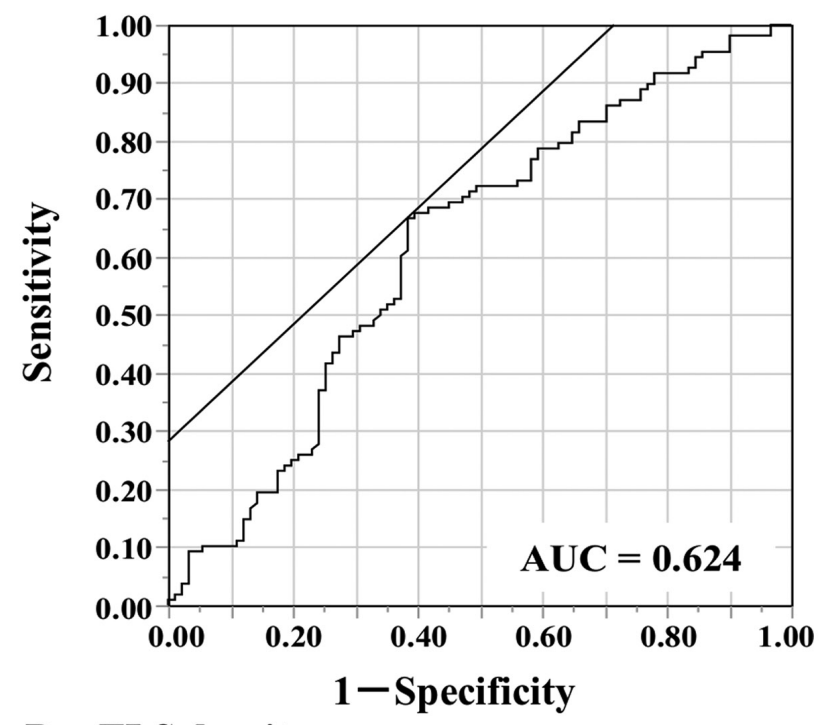

B TLS density

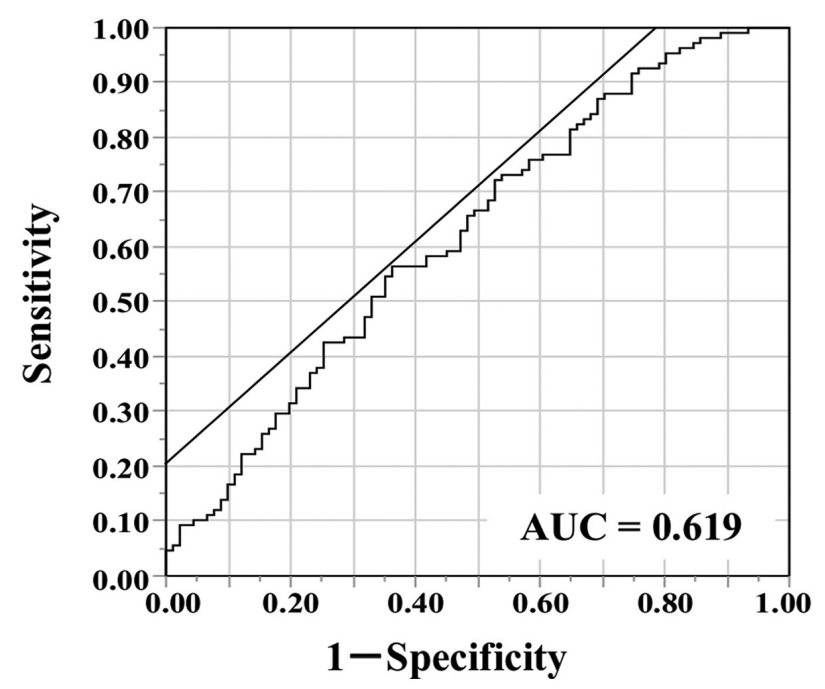

Figure 2. Receiver operating characteristic curves were used for determining the cut-off values. (A) Preoperative NLR and (B) TLS density. NLR, neutrophil-to-lymphocyte ratio; TLS, tertiary lymphoid structure; AUC, area under the curve.

were independently associated with the overall survival rate (Table III).

\section{Discussion}

In the present study, we investigated the relationships between the preoperative NLR and TLSs in the tumor. We showed that the NLR was potentially correlated with the TLS density, and both the NLR and TLSs were independent prognostic factors. Our results suggested that the systemic NLR might reflect the TLS density in the tumor microenvironment.

Tumor-associated neutrophils (TANs) exhibit plasticity and are capable of polarization into either an anti-tumorigenic ' $N 1$ ' phenotype or a pro-tumorigenic ' $N 2$ ' phenotype $(31,32)$. The 'N2' phenotype produces pro-tumorigenic factors, including vascular endothelial growth factor, inflammatory mediators and matrix metalloproteinases, and promotes tumor growth 
Table I. Association of the preoperative NLR with the clinicopathological characteristics of patients with gastric cancer ( $\mathrm{n}=199)$.

\begin{tabular}{|c|c|c|c|c|}
\hline Characteristics & No. of patients & NLR low $(n=108)$ & NLR high $(n=91)$ & P-value \\
\hline \multicolumn{5}{|l|}{ Age, years } \\
\hline$<60$ & 39 & 22 & 17 & \\
\hline$\geq 60$ & 160 & 86 & 74 & 0.7649 \\
\hline \multicolumn{5}{|l|}{ Sex } \\
\hline Male & 143 & 78 & 65 & \\
\hline Female & 56 & 30 & 26 & 0.9013 \\
\hline \multicolumn{5}{|l|}{ Smoking history } \\
\hline No & 122 & 61 & 61 & \\
\hline Yes & 77 & 47 & 30 & 0.1279 \\
\hline \multicolumn{5}{|l|}{ pT category } \\
\hline $\mathrm{T} 1$ & 15 & 11 & 4 & \\
\hline $\mathrm{T} 2$ & 48 & 26 & 22 & \\
\hline T3 & 45 & 29 & 16 & \\
\hline $\mathrm{T} 4$ & 91 & 42 & 49 & 0.0901 \\
\hline \multicolumn{5}{|l|}{ pN category } \\
\hline No & 65 & 39 & 26 & \\
\hline $\mathrm{N} 1-\mathrm{N} 3$ & 134 & 69 & 65 & 0.2586 \\
\hline \multicolumn{5}{|l|}{ pStage } \\
\hline $\mathrm{Ib}$ & 38 & 21 & 17 & \\
\hline II & 58 & 37 & 21 & \\
\hline III & 72 & 39 & 33 & \\
\hline IV & 31 & 11 & 20 & 0.0880 \\
\hline \multicolumn{5}{|l|}{ Histological type } \\
\hline tub1, tub2, pap & 82 & 50 & 32 & \\
\hline por, sig, muc & 115 & 57 & 58 & \\
\hline Others ${ }^{\mathrm{a}}$ & 2 & 1 & 1 & 0.2828 \\
\hline \multicolumn{5}{|c|}{ Lymphatic invasion } \\
\hline Negative & 33 & 24 & 9 & \\
\hline Positive & 166 & 84 & 82 & 0.0198 \\
\hline \multicolumn{5}{|l|}{ Venous invasion } \\
\hline Negative & 144 & 80 & 64 & \\
\hline Positive & 55 & 28 & 27 & 0.5563 \\
\hline
\end{tabular}

${ }^{a}$ Adenosquamous carcinoma and endocrine cell carcinoma. NLR, neutrophil-to-lymphocyte ratio; p, pathological; T, tumor; N, node; tub1, well differentiated tubular adenocarcinoma; tub2, moderately differentiated tubular adenocarcinoma; pap, papillary adenocarcinoma; por, poorly differentiated adenocarcinoma; sig, signet-ring cell carcinoma; muc, mucinous adenocarcinoma.

Table II. Association between the preoperative NLR and TLS density in the tumor microenvironment.

TLS density No. of patients NLR low NLR high P-value

\begin{tabular}{lrrrr}
\hline All cases & 199 & 108 & 91 & \\
TLS low & 105 & 45 & 60 & \\
TLS high & 94 & 63 & 31 & 0.0006 \\
\hline
\end{tabular}

NLR, neutrophil-to-lymphocyte ratio; TLS, tertiary lymphoid structure.

and progression (33). We previously reported that an increase in neutrophils at the tumor site was found within the primary tumor and lymph node metastasis, with a poor prognosis in patients with a high neutrophil count (34). Furthermore, we demonstrated experimentally that neutrophils exhibited an increased PD-L1 expression when they reacted with cancer cells and exerted an immunosuppressive function, such as the suppression of $\mathrm{T}$ cell proliferation by TANs (35).

The NLR is a systemic inflammation marker reported that has been to be an independent prognostic factor for survival in several malignancies (36-40). Among the many reports on gastric cancer, several studies have reported that the NLR may be a useful marker not only for surgery but also for chemotherapy and metastasis (41-45). In this study, we showed that the preoperative NLR was an independent prognostic factor for overall survival in gastric cancer patients, suggesting that an elevated NLR might reflect the host immune status. We previously reported that TANs were associated with tumor progression, and that high TAN infiltration was correlated with the preoperative NLR (46), with TANs exerting an immunosuppressive function (35). On the other hand, Choi et al showed that, within the tumor microenvironment, 
Table III. Results of the univariate and multivariate analyses of the prognostic factors of the overall survival for patients with gastric cancer.

\begin{tabular}{|c|c|c|c|c|}
\hline \multirow[b]{2}{*}{ Variables } & \multicolumn{2}{|c|}{ Univariate analysis } & \multicolumn{2}{|c|}{ Multivariate analysis } \\
\hline & HR $(95 \% \mathrm{CI})$ & P-value & $\mathrm{HR}(95 \% \mathrm{CI})$ & P-value \\
\hline Age $(<60 / \geq 60$ years $)$ & $1.273(0.762-2.265)$ & 0.3693 & NA & NA \\
\hline Sex (male/female) & $1.111(0.687-1.795)$ & 0.6653 & NA & NA \\
\hline pT category $(\mathrm{T} 1+\mathrm{T} 2 / \mathrm{T} 3+\mathrm{T} 4)$ & $3.153(1.866-5.708)$ & $<0.0001$ & $2.7(1.552-5.014)$ & 0.0002 \\
\hline $\mathrm{pN}$ category $(\mathrm{N} 0 / \mathrm{N} 1-3)$ & $2.423(1.492-4.134)$ & 0.0002 & $1.627(0.979-2.844)$ & 0.0609 \\
\hline pStage $(\mathrm{Ib}+\mathrm{II} / \mathrm{III}+\mathrm{IV})$ & $3.328(2.142-5.315)$ & $<0.0001$ & NA & NA \\
\hline Histological type (tub1,tub2,pap/por,sig,muc) & $1.809(1.171-2.848)$ & 0.0071 & $1.569(1.016-2.479)$ & 0.0422 \\
\hline Lymphatic invasion (negative/positive) & $7.629(2.861-31.081)$ & $<0.0001$ & $2.901(0.992-12.367)$ & 0.0520 \\
\hline Venous invasion (negative/positive) & $1.092(0.676-1.710)$ & 0.7102 & NA & NA \\
\hline NLR (low/high) & $2.300(1.516-3.531)$ & $<0.0001$ & $1.65(1.068-2.579)$ & 0.0241 \\
\hline TLS (high/low) & $2.003(1.314-3.104)$ & 0.0012 & $2.042(1.311-3.227)$ & 0.0015 \\
\hline
\end{tabular}

HR, hazard ratio; NLR, neutrophil-to-lymphocyte ratio; TLS, tertiary lymphoid structure; NA, not applicable; p, pathological; T, tumor; $\mathrm{N}$, node; tub1, well differentiated tubular adenocarcinoma; tub2, moderately differentiated tubular adenocarcinoma; pap, papillary adenocarcinoma; por, poorly differentiated adenocarcinoma; sig, signet-ring cell carcinoma; muc, mucinous adenocarcinoma.

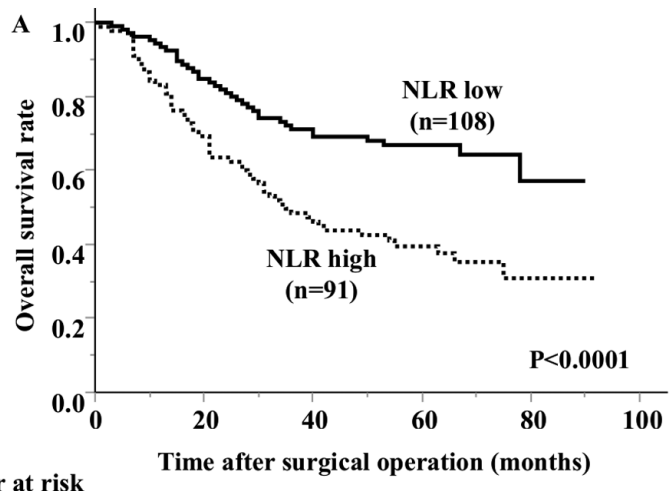

Number at risk

$\begin{array}{ccccccc}\text { NLR low } & 91 & 90 & 70 & 47 & 6 & 0 \\ \text { NLR high } & 108 & 61 & 40 & 25 & 5 & 0\end{array}$

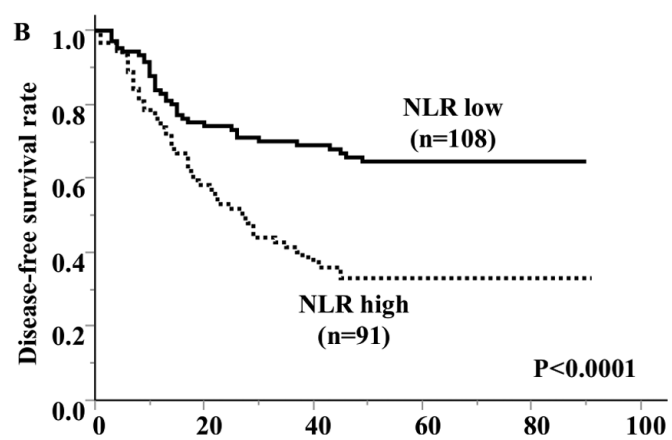

Number at risk

Time after surgical operation (months)

$\begin{array}{ccccccc}\text { NLR low } & 91 & 73 & 65 & 42 & 7 & 0 \\ \text { NLR high } & 108 & 46 & 28 & 19 & 3 & 0\end{array}$

Figure 3. Kaplan-Meier survival curves stratified according to preoperative NLR levels. (A) Overall survival and (B) disease-free survival. NLR, neutrophilto-lymphocyte ratio.

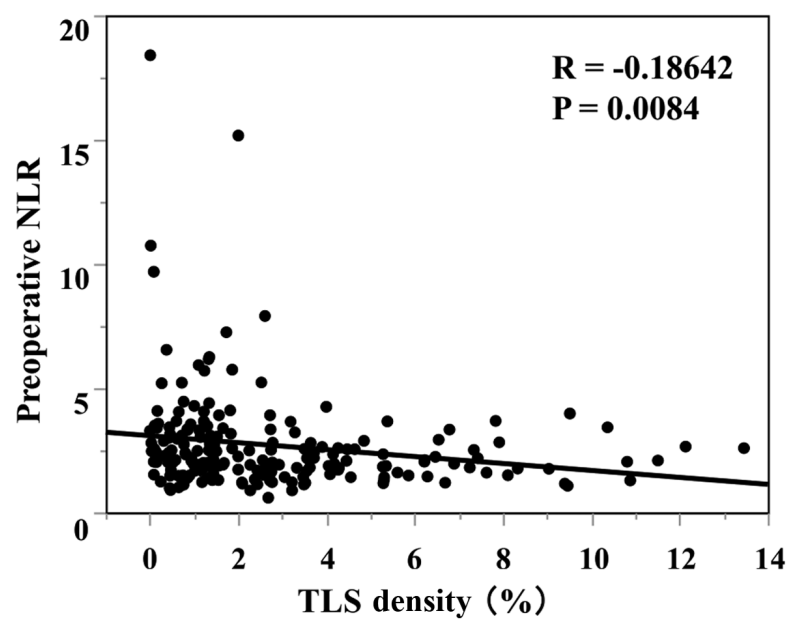

Figure 4. Correlation between the preoperative NLR and TLS density in the tumor microenvironment. NLR, neutrophil-to-lymphocyte ratio; TLS, tertiary lymphoid structure. the NLR was associated with the density of $\mathrm{CD}^{+} \mathrm{T}$ cells, supporting the prognostic value of systemic inflammation in gastric cancer (47). Tanaka et al also showed that in biliary tract cancer, the preoperative NLR was negatively correlated with $\mathrm{CD}^{+}$TILs, and that it may predict CD8 ${ }^{+}$TILs infiltrating in the tumor microenvironment (48). Furthermore, it has been reported that the high pre-treatment NLR was significantly associated with high neutrophil infiltration and low $\mathrm{CD}^{+} \mathrm{T}$ cell infiltration into tumors in patients with glioblastoma (49), and that the preoperative NLR might originate from proinflammatory conditions such as tumor necrosis or absence of TILs in hepatocellular carcinoma (50).

ICB to unleash an antitumor immune response results in a durable effect in gastric cancer (51). However, because some patients do not respond to ICB therapy, case selection will be necessary in the future. Increased local antitumor immune mechanisms, or TILs, are thought to be hot tumors and are more responsive to ICB therapy. An analysis of samples from 


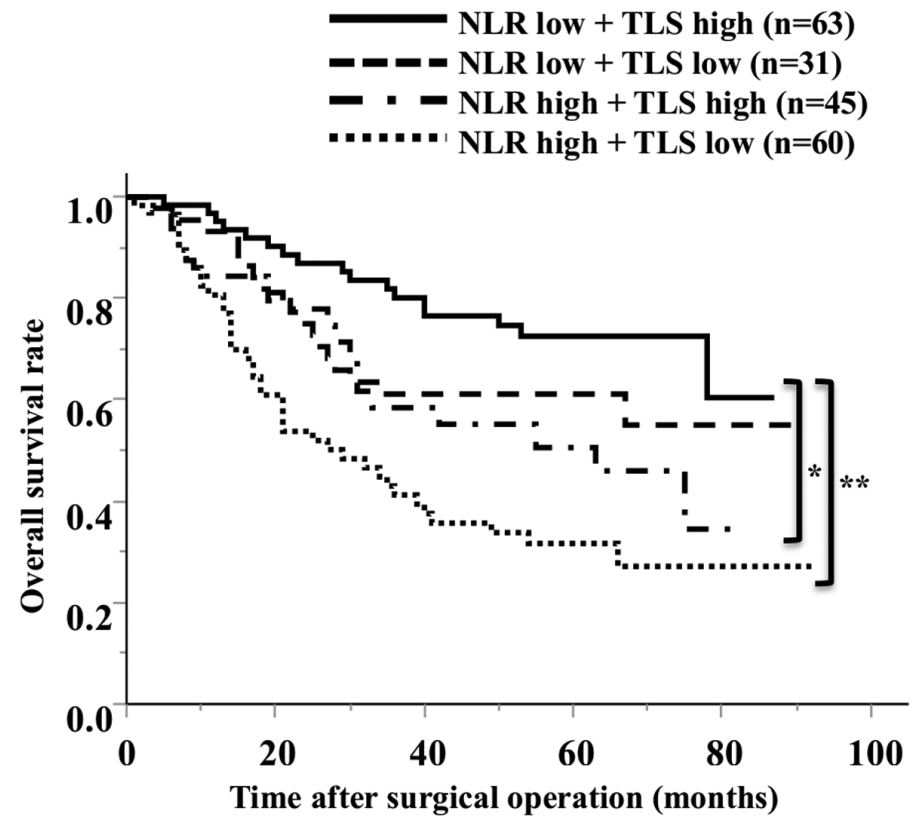

Figure 5. Overall survival rates according to the combination of the preoperative NLR and TLS density. ${ }^{*} \mathrm{P}=0.0102 ;{ }^{* *} \mathrm{P}<0.001$. NLR, neutrophil-to-lymphocyte ratio; TLS, tertiary lymphoid structure.

A Stage Ib

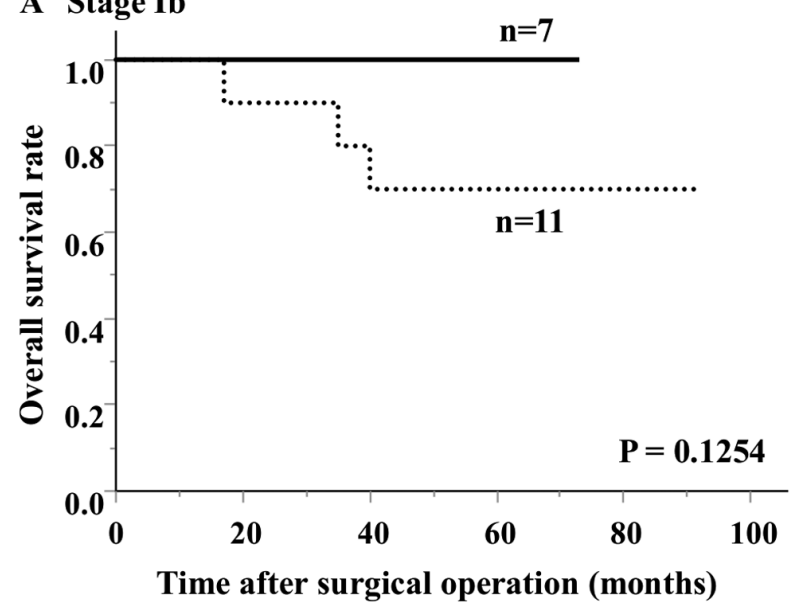

C Stage III

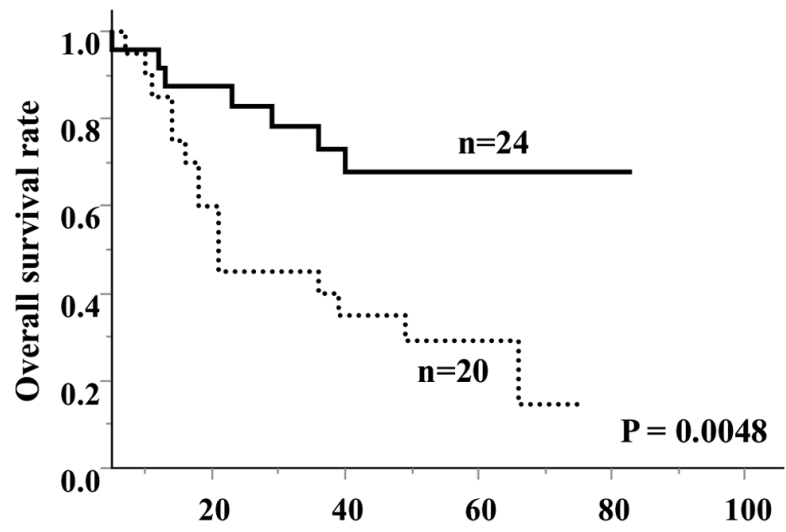

Time after surgical operation (months)
B Stage II

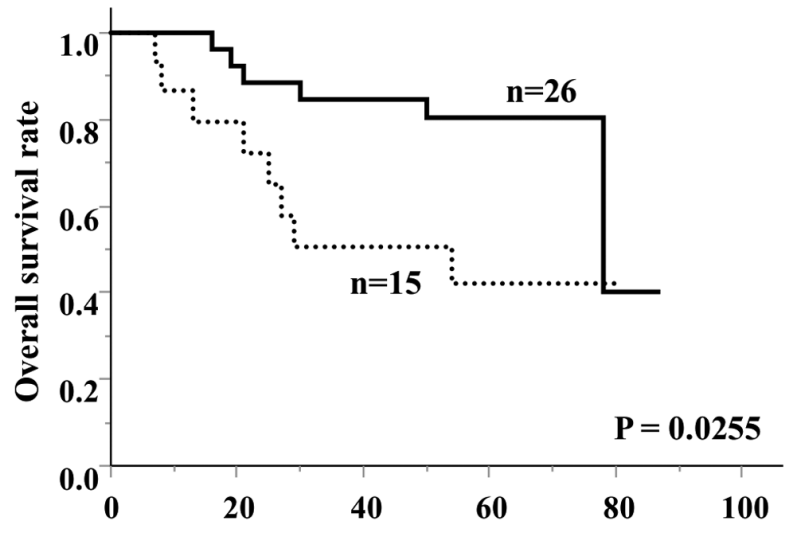

Time after surgical operation (months)

D Stage IV

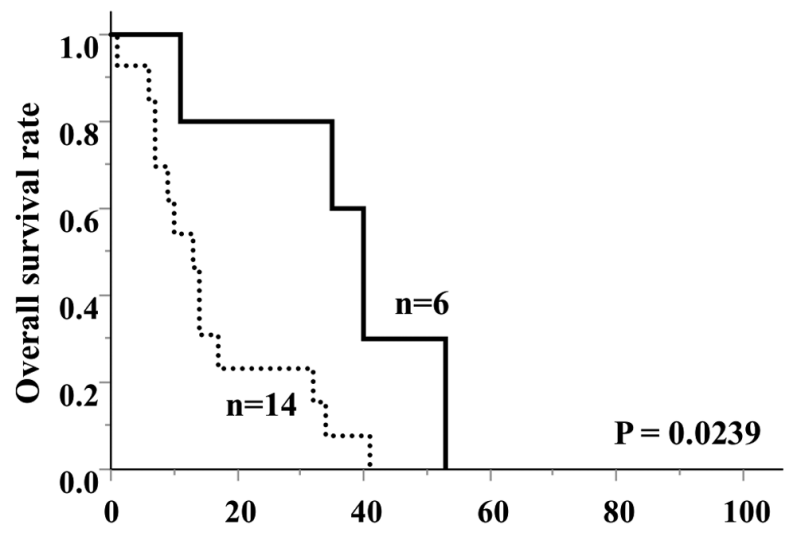

Time after surgical operation (months)

Figure 6. Analysis of the overall survival rates at different tumor stages according to the combination of the preoperative NLR and TLS density. (A) Stage Ib, (B) stage II, (C) stage III and (D) stage IV. NLR, neutrophil-to-lymphocyte ratio; TLS, tertiary lymphoid structure. 
clinical trials of ICB-treated malignant melanoma and renalcell carcinoma cases reported that the maturity of B cells in TLSs was associated with the treatment response (12). We previously reported the presence of TLSs in gastric cancer tissue, the correlation of peri-tumor TLSs with TILs, and the favorable prognosis of TLSs observed more frequently in patients with gastric cancer (11). In addition, we showed that an analysis of B cells in TLSs was able to induce CTLs in TLSs and TLSs may be involved in cellular immunity (52). TLSs can also be associated with humoral immunity (53). These results suggest that TLSs plays an important role in the induction of local tumor immunity. Moreover, in this study, we suggested a potential association between the NLR and TLSs in the tumor microenvironment.

The present findings suggest that the NLR may be useful for evaluating the immunologic status of the tumor microenvironment. Importantly, the NLR can be easily calculated from peripheral blood counts, eliminating the need for invasive procedures, such as a tissue biopsy, to evaluate the tumor microenvironment. Thus, the NLR in the peripheral blood might serve as an easy and useful marker for evaluating the immunoreactivity in the tumor microenvironment.

The present study was associated with some limitations. First, this was a retrospective, single-center study with a relatively small number of patients. Thus, the results may be biased. Second, the correlation between the preoperative NLR and TLS density did not reach statistical significance. Further studies, including prospective studies with a larger number of patients, should be performed to confirm our findings.

In conclusion, the preoperative NLR appears to be correlated with the TLS density in the primary tumor and to be a useful prognostic factor. Our results suggested that the local immune response might be related to systemic neutrophilic induction. Therefore, the preoperative NLR and TLSs surrounding the tumor may be a predictive biomarker with applications in cancer immunotherapy against gastric cancer.

\section{Acknowledgements}

Not applicable.

\section{Funding}

No funding was received.

\section{Availability of data and materials}

The datasets used and/or analyzed in the current study are available from the corresponding author on reasonable request.

\section{Authors' contributions}

YY acquired, analyzed and interpreted the data, confirmed the authenticity of the data and drafted the manuscript. HT made substantial contributions to the conception and design of the study, interpreted the data, confirmed the authenticity of the data and revised the manuscript critically. CS, TM, SD,
MY, TTa, TTo, SL and KM acquired and analyzed the data. $\mathrm{KH}$ and $\mathrm{MO}$ contributed to the conception and design of the study, and revised the manuscript critically. All authors read and approved the final manuscript.

\section{Ethics approval and consent to participate}

All experimental procedures after 2013 were approved by the Osaka City University Ethics Committee (approval no. 3138; Osaka, Japan), and all patients provided written informed consent for the collection and analysis of the specimens. All patients who were managed prior to 2013 provided their written informed consent for sample collection and were allowed to withdraw from the study by signing an opt-out form approved by the Osaka City University Ethics Committee (approval no. 4092).

\section{Patient consent for publication}

Not applicable.

\section{Competing interests}

The authors declare that they have no competing interests.

\section{References}

1. Figueroa-Protti L, Soto-Molinari R, Calderon-Osorno M, Mora J and Alpizar-Alpizar W: Gastric cancer in the Era of immune checkpoint blockade. J Oncol 2019: 1079710, 2019.

2. Al-Shibli KI, Donnem T, Al-Saad S, Persson M, Bremnes RM and Busund LT: Prognostic effect of epithelial and stromal lymphocyte infiltration in non-small cell lung cancer. Clin Cancer Res 14: 5220-5227, 2008

3. Sudo T, Nishida R, Kawahara A, Saisho K, Mimori K, Yamada A, Mizoguchi A, Kadoya K, Matono S, Mori N, et al: Clinical impact of tumor-infiltrating lymphocytes in esophageal squamous cell carcinoma. Ann Surg Oncol 24: 3763-3770, 2017.

4. Kang BW, Seo AN, Yoon S, Bae HI, Jeon SW, Kwon OK, Chung HY, Yu W, Kang $\mathrm{H}$ and Kim JG: Prognostic value of tumor-infiltrating lymphocytes in Epstein-Barr virus-associated gastric cancer. Ann Oncol 27: 494-501, 2016.

5. Kong JC, Guerra GR, Pham T, Mitchell C, Lynch AC, Warrier SK, Ramsay RG and Heriot AG: Prognostic impact of tumor-infiltrating lymphocytes in primary and metastatic colorectal cancer: A systematic review and meta-analysis. Dis Colon Rectum 62: 498-508, 2019.

6. Stanton SE and Disis ML: Clinical significance of tumor-infiltrating lymphocytes in breast cancer. J Immunother Cancer 4: 59, 2016.

7. Germain C, Gnjatic S, Tamzalit F, Knockaert S, Remark R, Goc J, Lepelley A, Becht E, Katsahian S, Bizouard G, et al: Presence of $\mathrm{B}$ cells in tertiary lymphoid structures is associated with a protective immunity in patients with lung cancer. Am J Respir Crit Care Med 189: 832-844, 2014.

8. Hennequin A, Derangere V, Boidot R, Apetoh L, Vincent J, Orry D, Fraisse J, Causeret S, Martin F, Arnould L, et al: Tumor infiltration by Tbet $^{+}$effector T cells and CD $20^{+} \mathrm{B}$ cells is associated with survival in gastric cancer patients. Oncoimmunology 5: e1054598, 2016.

9. Schweiger T, Berghoff AS, Glogner C, Glueck O, Rajky O, Traxler D, Birner P, Preusser M, Klepetko W and Hoetzenecker K: Tumor-infiltrating lymphocyte subsets and tertiary lymphoid structures in pulmonary metastases from colorectal cancer. Clin Exp Metastasis 33: 727-739, 2016.

10. Figenschau SL, Fismen S, Fenton KA, Fenton C and Mortensen ES: Tertiary lymphoid structures are associated with higher tumor grade in primary operable breast cancer patients. BMC Cancer 15: 101, 2015.

11. Sakimura C, Tanaka H, Okuno T, Hiramatsu S, Muguruma K, Hirakawa K, Wanibuchi $\mathrm{H}$ and Ohira M: B cells in tertiary lymphoid structures are associated with favorable prognosis in gastric cancer. J Surg Res 215: 74-82, 2017. 
12. Helmink BA, Reddy SM, Gao J, Zhang S, Basar R, Thakur R, Yizhak K, Sade-Feldman M, Blando J, Han G, et al: B cells and tertiary lymphoid structures promote immunotherapy response. Nature 577: 549-555, 2020.

13. CabritaR,Lauss M,Sanna A,Donia M,SkaarupLarsen M,Mitra S, Johansson I, Phung B, Harbst K, Vallon-Christersson J, et al: Tertiary lymphoid structures improve immunotherapy and survival in melanoma. Nature 577: 561-565, 2020.

14. Sautès-Fridman C, Lawand M, Giraldo NA, Kaplon H, Germain C, Fridman WH and Dieu-Nosjean MC: Tertiary lymphoid structures in cancers: Prognostic value, regulation, and manipulation for therapeutic intervention. Front Immunol 7: 407, 2016.

15. Pimenta EM and Barnes BJ: Role of tertiary lymphoid structures (TLS) in anti-tumor immunity: Potential tumor-induced cytokines/chemokines that regulate TLS formation in epithelialderived cancers. Cancers (Basel) 6: 969-997, 2014.

16. Carragher DM, Rangel-Moreno J and Randall TD: Ectopic lymphoid tissues and local immunity. Semin Immunol 20: 26-42, 2008.

17. Nagtegaal ID, Marijnen CA, Kranenbarg EK, Mulder-Stapel A Hermans J, van de Velde CJ and van Krieken JH: Local and distant recurrences in rectal cancer patients are predicted by the nonspecific immune response; specific immune response has only a systemic effect-a histopathological and immunohistochemical study. BMC Cancer 1: 7, 2001.

18. Guner A and Kim HI: Biomarkers for evaluating the inflammation status in patients with cancer. J Gastric Cancer 19: 254-277, 2019.

19. Kosumi K, Baba Y, Ishimoto T, Harada K, Nakamura K, Ohuchi M, Kiyozumi Y, Izumi D, Tokunaga R, Taki K, et al: Neutrophil/lymphocyte ratio predicts the prognosis in esophageal squamous cell carcinoma patients. Surg Today 46: 405-413, 2016.

20. Zhang X, Zhang W and Feng LJ: Prognostic significance of neutrophil lymphocyte ratio in patients with gastric cancer: A meta-analysis. PLoS One 9: e111906, 2014.

21. Haram A, Boland MR, Kelly ME, Bolger JC, Waldron RM and Kerin MJ: The prognostic value of neutrophil-to-lymphocyte ratio in colorectal cancer: A systematic review. J Surg Oncol 115 470-479, 2017.

22. Gao F, Li X, Geng M, Ye X, Liu H, Liu Y, Wan G and Wang X Pretreatment neutrophil-lymphocyte ratio: An independent predictor of survival in patients with hepatocellular carcinoma. Medicine (Baltimore) 94: e639, 2015.

23. Mowbray NG, Griffith D, Hammoda M, Shingler G, Kambal A and Al-Sarireh B: A meta-analysis of the utility of the neutrophil-to-lymphocyte ratio in predicting survival after pancreatic cancer resection. HPB (Oxford) 20: 379-384, 2018.

24. Aktas G, Sit M, Dikbas O, Erkol H, Altinordu R, Erkus E and Savli H: Elevated neutrophil-to-lymphocyte ratio in the diagnosis of Hashimoto's thyroiditis. Rev Assoc Med Bras (1992) 63 1065-1068, 2017

25. Sit M, Aktas G, Erkol H, Yaman S, Keyif F and Savli H: Neutrophil to lymphocyte ratio is useful in differentiation of malign and benign thyroid nodules. P R Health Sci J 38: 60-63, 2019.

26. Duman TT, Aktas G, Atak BM, Kocak MZ, Erkus E and Savli H: Neutrophil to lymphocyte ratio as an indicative of diabetic control level in type 2 diabetes mellitus. African Health Sci 19: 1602-1606, 2019.

27. Bilgin S, Aktas G, Zahid Kocak M, Atak BM, Kurtkulagi O, Duman TT and Savli H: Association between novel inflammatory markers derived from hemogram indices and metabolic parameters in type 2 diabetic men. Aging Male: 1-5, 2019.

28. Jeong Y, Jeon SR, Kim HG, Moon JR, Lee TH, Jang JY, Cho JH, Park JS, Park H, Lee KH, et al: The role of platelet to lymphocyte ratio and neutrophil to lymphocyte ratio in ulcerative colitis. Intest Res 19: 62-70, 2021.

29. Aktas G, Duman T, Atak B, Kurtkulagi O, Bilgin S, Basaran E, Demirkol ME and Kosekli MA: Irritable bowel syndrome is associated with novel inflammatory markers derived from hemogram parameters. Fam Med Prim Care Rev 22: 107-110, 2020.

30. Khoury T, Mari A, Nseir W, Kadah A, Sbeit W and Mahamid M: Neutrophil-to-lymphocyte ratio is independently associated with inflammatory activity and fibrosis grade in nonalcoholic fatty liver disease. Eur J Gastroenterol Hepatol 31: 1110-1115, 2019.

31. Fridlender ZG, Sun J, Kim S, Kapoor V, Cheng G, Ling L, Worthen GS and Albelda SM: Polarization of tumor-associated neutrophil phenotype by TGF-beta: 'N1' versus 'N2' TAN. Cancer Cell 16: 183-194, 2009.
32. Mishalian I, Bayuh R, Levy L, Zolotarov L, Michaeli J and Fridlender ZG: Tumor-associated neutrophils (TAN) develop pro-tumorigenic properties during tumor progression. Cancer Immunol Immunother 62: 1745-1756, 2013

33. Hurt B, Schulick R, Edil B, El Kasmi KC and Barnett C Jr: Cancer-promoting mechanisms of tumor-associated neutrophils. Am J Surg 214: 938-944, 2017

34. Tokumoto M, Tanaka H, Ohira M, Go Y, Okita Y, Sakurai K, Toyokawa T, Kubo N, Muguruma K, Maeda K, et al: A positive correlation between neutrophils in regional lymph nodes and progression of gastric cancer. Anticancer Res 34: 7129-7136, 2014.

35. Hiramatsu S, Tanaka H, Nishimura J, Yamakoshi Y, Sakimura C, Tamura T, Toyokawa T, Muguruma K, Yashiro M Hirakawa K and Ohira M: Gastric cancer cells alter the immunosuppressive function of neutrophils. Oncol Rep 43: 251-259, 2020.

36. Nakamura K, Yoshida N, Baba Y, Kosumi K, Uchihara T, Kiyozumi Y, Ohuchi M, Ishimoto T, Iwatsuki M, Sakamoto Y, et al: Elevated preoperative neutrophil-to-lymphocytes ratio predicts poor prognosis after esophagectomy in $\mathrm{T} 1$ esophageal cancer. Int J Clin Oncol 22: 469-475, 2017.

37. Song Y, Yang Y, Gao P, Chen X, Yu D, Xu Y, Zhao J and Wang Z: The preoperative neutrophil to lymphocyte ratio is a superior indicator of prognosis compared with other inflammatory biomarkers in resectable colorectal cancer. BMC Cancer 17: 744 2017.

38. Fu Y, Liu W, OuYang D, Yang A and Zhang Q: Preoperative neutrophil-to-lymphocyte ratio predicts long-term survival in patients undergoing total laryngectomy with advanced laryngeal squamous cell carcinoma: A single-center retrospective study. Medicine (Baltimore) 95: e2689, 2016.

39. Feng Z, Wen H, Bi R, Ju X, Chen X, Yang W and Wu X: Preoperative neutrophil-to-lymphocyte ratio as a predictive and prognostic factor for high-grade serous ovarian cancer. PLoS One 11: e0156101, 2016

40. Nayak A, McDowell DT, Kellie SJ and Karpelowsky J: Elevated preoperative neutrophil-lymphocyte ratio is predictive of a poorer prognosis for pediatric patients with solid tumors. Ann Surg Oncol 24: 3456-3462, 2017.

41. Mori M, Shuto K, Kosugi C, Narushima K, Hayashi H, Matsubara $\mathrm{H}$ and Koda K: An increase in the neutrophil-tolymphocyte ratio during adjuvant chemotherapy indicates a poor prognosis in patients with stage II or III gastric cancer. BMC Cancer 18: 1261, 2018.

42. Pang W, Lou N, Jin C, Hu C, Arvine C, Zhu G and Shen X: Combination of preoperative platelet/lymphocyte and neutrophil/lymphocyte rates and tumor-related factors to predict lymph node metastasis in patients with gastric cancer. Eur J Gastroenterol Hepatol 28: 493-502, 2016.

43. Nakayama Y, Gotohda N, Shibasaki H, Nomura S, Kinoshita T and Hayashi R: Usefulness of the neutrophil/lymphocyte ratio measured preoperatively as a predictor of peritoneal metastasis in patients with advanced gastric cancer. Surg Today 44: 21462152,2014

44. Tanaka H, Tamura T, Toyokawa T, Muguruma K, Miki Y, Kubo N, Sakurai K, Hirakawa K and Ohira M: Clinical relevance of postoperative neutrophil-lymphocyte ratio (NLR) to recurrence after adjuvant chemotherapy of S-1 for gastric cancer. Anticancer Res 38: 3745-3751, 2018.

45. Tanaka H, Muguruma K, Toyokawa T, Kubo N, Ohira M and Hirakawa K: Differential impact of the neutrophil-lymphocyte ratio on the survival of patients with stage IV gastric cancer. Dig Surg 31: 327-333, 2014.

46. Hiramatsu S, Tanaka H, Nishimura J, Sakimura C, Tamura T, Toyokawa T, Muguruma K, Yashiro M, Hirakawa K and Ohira M: Neutrophils in primary gastric tumors are correlated with neutrophil infiltration in tumor-draining lymph nodes and the systemic inflammatory response. BMC Immunol 19: 13, 2018

47. Choi Y, Kim JW, Nam KH, Han SH, Kim JW, Ahn SH, Park DJ, Lee KW, Lee HS and Kim HH: Systemic inflammation is associated with the density of immune cells in the tumor microenvironment of gastric cancer. Gastric Cancer 20: 602-611, 2017

48. Tanaka R, Kimura K, Eguchi S, Tauchi J, Shibutani M, Shinkawa H, Ohira GO, Yamazoe S, Tanaka S, Amano R, et al: Preoperative neutrophil-to-lymphocyte ratio predicts tumorinfiltrating CD8(+) T cells in biliary tract cancer. Anticancer Res 40: 2881-2887, 2020 
49. Ha SY, Choi S, Park S, Kim JM, Choi GS, Joh JW and Park CK Prognostic effect of preoperative neutrophil-lymphocyte ratio is related with tumor necrosis and tumor-infiltrating lymphocytes in hepatocellular carcinoma. Virchows Archiv 477: 807-816, 2020.

50. Han S, Liu Y, Li Q, Li Z, Hou H and Wu A: Pre-treatment neutrophil-to-lymphocyte ratio is associated with neutrophil and T-cell infiltration and predicts clinical outcome in patients with glioblastoma. BMC Cancer 15: 617, 2015.

51. Kang YK, Boku N, Satoh T, Ryu MH, Chao Y, Kato K, Chung HC, Chen JS, Muro K, Kang WK, et al: Nivolumab in patients with advanced gastric or gastro-oesophageal junction cancer refractory to, or intolerant of, at least two previous chemotherapy regimens (ONO-4538-12, ATTRACTION-2): A randomised, double-blind, placebo-controlled, phase 3 trial. Lancet 390: 2461-2471, 2017.
52. Yamakoshi Y, Tanaka H, Sakimura C, Deguchi S, Mori T, Tamura T, Toyokawa T, Muguruma K, Hirakawa K and Ohira M: Immunological potential of tertiary lymphoid structures surrounding the primary tumor in gastric cancer. Int J Oncol 57: 171-182, 2020.

53. Sautès-Fridman C, Petitprez F, Calderaro J and Fridman WH: Tertiary lymphoid structures in the era of cancer immunotherapy. Nat Rev Cancer 19: 307-325, 2019.

(i) (9) This work is licensed under a Creative Commons EY No No Attribution-NonCommercial-NoDerivatives 4.0 International (CC BY-NC-ND 4.0) License. 